\title{
Digital Ischaemia following Radial Arterial Cannulation
}

\author{
Alison Deasy ${ }^{1}$, Tanya O’Neill ${ }^{1}$, Daniel Rawluk ${ }^{2}$, and Joseph Deasy ${ }^{2}$ \\ ${ }^{1}$ Beaumont Hospital Department of Anaesthesia \\ ${ }^{2}$ Beaumont Hospital
}

February 26, 2021

\begin{abstract}
Indwelling arterial catheters are used routinely for continuous haemodynamic monitoring during major surgery and in critically ill patients. Major complications are rare. We describe two cases of patients in which arterial catheterisation was associated with acute radial arterial thrombosis and radial ischaemic neuropathy. Timely recognition was paramount to successful management.
\end{abstract}

Digital Ischaemia following Radial Arterial Cannulation

Alison Deasy, Beaumont Hospital, Dublin, Ireland ${ }^{1}$ *

Corresponding Author. Email : alisondeasy@rcsi.ie

T. O'Neill, Beaumont Hospital, Dublin, Ireland ${ }^{2}$

D. Rawluk, Beaumont Hospital, Dublin, Ireland ${ }^{3}$

J. Deasy, Beaumont Hospital, Dublin, Ireland ${ }^{4}$

INTRODUCTION:

Indwelling arterial catheters are used routinely for continuous haemodynamic monitoring and for obtaining repetitive blood samples during major surgery and in critically ill patients. The radial artery is the most common site for catheterisation. Major complications such as ischaemic insult, sepsis and pseudoaneurysm formation are rare, occurring in fewer than $1 \%$ of cases(1). We describe two cases of patients undergoing major surgical and endovascular interventions in which arterial catheterisation was associated with acute radial arterial thrombosis and radial ischaemic neuropathy.

CASE PRESENTATION - PATIENT 1:

An 88-year old female was scheduled for a low anterior resection and end-colostomy formation for a high grade invasive recto-sigmoid adenocarcinoma. She had suffered an acute kidney injury attributable to the mass effect of the tumour involving the bladder that required a rigid cystoscopy and stent insertion prior to laparotomy. Her past medical history included low body mass index, shingles, recurrent urinary tract infection, uterine prolapse and a single functioning kidney. Prior to her admission, she lived at home alone and was fully independent. She did not take any regular medications and had no known drug allergies. Her pre-operative bloods results demonstrated an iron-deficiency anaemia (Haemoglobin $10 \mathrm{~g} / \mathrm{dL}$ ), acute kidney injury and raised inflammatory markers consistent with a contained colonic perforation. Coagulation studies were normal. She had been receiving a daily prophylactic dose of enoxaparin $(20 \mathrm{mg})$ in the preoperative period.

Prior to induction of anaesthesia, a 20G arterial catheter (Arterial Leader Cath (PE), Vygon Ref 115.090, 3Fr- L. $8 \mathrm{~cm}-0.9 \mathrm{~mm}-24 \mathrm{ml} / \mathrm{min}$ ) was inserted in the left radial artery using the Seldinger technique. The 
first attempt was successful and the arterial catheter was introduced without resistance. Blood could be withdrawn and a normal pulse tracing was shown on the monitor. No discoloration of the hand was noted. General anaesthesia was initiated uneventfully and the patient was positioned on the surgical table in lithotomy with all necessary padding and safety straps applied. All pressure areas including venous and arterial lines were padded and protected. There were no intra-operative issues with the arterial catheter. The surgery concluded after approximately five hours without any complications and minimal estimated blood loss.

At the end of the procedure, progressive dampening of the arterial line trace was noted on the monitor and blood could not be aspirated through it. On removal of the surgical drapes, the left hand was noted to be cold and pale with a prolonged capillary refill time of five seconds. The arterial catheter was disconnected from the transducer tubing and there was evidence of thrombus lodged in the catheter. The catheter was promptly removed and the hand was actively warmed. The patient was extubated and transferred to the recovery room where an urgent consultation with a vascular surgeon was obtained. On further examination, sensation and motor function in the left hand were intact. Doppler ultrasound examination was unable to detect radial, ulnar or palmar arch pulsations adequately. The brachial pulse was detectable.

Following on immediate advice from vascular and colorectal surgical colleagues, treatment with a heparin infusion was not favoured following major abdominal surgery. CT angiography was contraindicated due to her acute kidney injury. Surgical intervention was deemed infeasible. After obtaining consent from the patient, a left-sided stellate ganglion block was performed by an experienced pain specialist. After two hours, the patient's hand colour and temperature improved with a commensurate reduction in capillary refill time. The patient was transferred to the high dependency unit for ongoing post-surgical observation and was reviewed by the vascular team on a daily basis thereafter.

Within 24 hours, doppler signals in the left radial and ulnar branches were detectable. The fourth and fifth digits remained cold to touch, with intact sensation, for a further 48 hours. Subsequently there was full resolution of symptoms with no requirement for further intervention.

\section{CASE PRESENTATION - PATIENT 2:}

A 66-year old female was scheduled for an elective endovascular coiling of her right posterior communicating artery aneurysm in the interventional radiology suite. This was an incidental finding during investigation of recurrent headaches. Her medical history included emphysema, ischaemic heart disease, dyslipidaemia, hypertension and osteoporosis. She was a current smoker with a 40-pack year history.

After induction of anaesthesia, a 20G arterial catheter (Arterial Leader Cath (PE), Vygon Ref 115.090, 3Fr- L. $8 \mathrm{~cm}-0.9 \mathrm{~mm}-24 \mathrm{ml} / \mathrm{min}$ ) was inserted in the left radial artery using the Seldinger technique. The first attempt was successful and the arterial catheter was introduced without resistance. Blood could be withdrawn and a normal pulse tracing was shown on the monitor. The coiling proceeded uneventfully without any complications. There were no intra- or post-operative issues with the arterial catheter, and it was removed in the recovery area prior to transfer to the ward.

The following day, the patient noted a gradual discoloration and reduced sensation in her left index, third and fourth digits. On examination, reduced temperature and sensation was noted with intact radial pulsation. Motor power was normal. An ultrasound scan of her left upper limb demonstrated patent left brachial and ulnar arteries, and greater than $50 \%$ stenosis of the left radial artery. The patient experienced progressive purple-bluish discoloration of the left index finger with increasing tenderness. After consultation with vascular surgery, she received a loading dose of 5,000 IU of intravenous Heparin and 100mcg of Iloprost. She continued on their respective infusions (dosing as per local guidelines) as well as oral dual anti-platelet therapies, Aspirin 75mg and Clopidogrel 75mg, once daily. Topical Glyceryl Trinitrate ointment was applied to the affected fingers for analgesic and vasodilatory purposes. Complete resolution of her symptoms was observed after eight days of treatment and she was discharged for follow up in vascular outpatient service.

\section{DISCUSSION:}

Easy access and low complication rates have resulted in the radial artery being the most popular site for 
arterial cannulation. The most common reported complication is temporary arterial occlusion with a mean incidence of $19.7 \%(1)$. Serious ischaemic injury resulting from permanent occlusion is, however, rare (mean incidence $0.09 \%$ ) but the potential consequences of necrosis and finger amputation are significant(1). The benefits of monitoring with arterial catheterisation are obvious but must be balanced against the associated risks and should outweigh the potential harms. It has been shown that the incidence of radial artery occlusion increases linearly with the ratio of outer diameter of the arterial catheter to vessel lumen diameter. This may explain the higher incidence in females and the preference for using narrow lumen catheters $(2,3)$. Other risk factors include low body mass index, advanced age, vascular disease, prolonged peri-procedural hypo- and/or hypertension, vasopressor use, catheter composition, prolonged catheter placement and excess trauma from multiple attempts at the same site $(4,5)$. It is possible that risk factors, such as low body mass index, advanced age, vascular disease and female gender, may have contributed to the temporary occlusion in both patients outlined above.

Radial artery occlusion (RAO) is the most frequent post-procedural complication of transradial access in cardiac catheterisation(6). This can have significant clinical implications, such as, precluding the use of the ipsilateral radial artery for future procedures and as a conduit for coronary artery bypass grafting or for arteriovenous fistula creation in patients requiring haemodialysis. Therefore, prevention is of upmost clinical importance. During radial haemostasis, complete cessation of blood flow with "occlusive" compression promotes thrombus formation and is a strong predictor of RAO (7). In the PROPHET trial, the 'patent' or nonocclusive haemostasis protocol, was compared with to conventional pressure application for haemostasis after transradial diagnostic coronary angiography. The patent haemostasis technique was associated with a significant decrease in early ( $<24$ hour) RAO rates from $12 \%$ to $5 \%$ and late RAO rates (30 days) from $7 \%$ to $1.8 \%$ (8). Similarly, in the RACOMAP trial, patent haemostasis was performed with a pneumatic compression device in which compression was guided by mean arterial pressure (maintaining thereby flow within the radial artery during haemostasis), demonstrating also a significant decrease in RAO rates from $12.0 \%$ to $1.1 \%$ (9). Despite being simple and inexpensive non-pharmacological method of preventing RAO, it requires significant involvement of the nursing staff due to the need for repeated oximetry-plethysmographic evaluation of radial flow and frequent adaptation of the haemostatic pressure to ensure ongoing vessel patency. As such, there is still limited adoption of the technique worldwide(10).

The consequences of RAO are generally benign when managed appropriately in a timely manner. A cold, pale hand or fingers with reduced motor function, sensory deficit or pain following radial artery cannulation should be immediately investigated. Management options include medical and/or surgical intervention. If ischaemia is suspected, the catheter should be removed immediately and the affected limb elevated and warmed. Anticoagulant, thrombolytic and vasodilator therapy may be indicated if there is a perceived risk of progressive ischaemia following these conservative measures. Some cardiology data suggests that immediate treatment with 5,000 IU intravenous heparin combined with compression of the ipsilateral ulnar artery for 1 hour or, alternatively, body-weight-adjusted therapeutic dosing of enoxaparin or fondaparinux for 4 weeks may help recanalise the radial artery(11). Some case reports have described successful recanalisation of the occluded radial artery with angioplasty $(12,13)$. In certain cases, thrombectomy, surgical bypass or cervical sympathetic blockage should be considered. However surgical intervention in the setting of iatrogenic radial arterial thrombosis is uncommon(14).

There is strong evidence for the use of ultrasound guidance in radial artery catheterisation in both adult and paediatric populations. Ultrasound guidance significantly increases first-attempt success rate, which subsequently results in a significant reduction in the number of attempts(15). The early use of ultrasound can be a valuable adjunct in radial arterial catheterisation and should be considered, especially in predicted difficult cases.

The Allen's test or modified Allen's test are bedside tests that can be performed in patients undergoing radial artery puncture. The hand is perfused by both the radial and ulnar arteries with extensive collateral flow between the two. However, some patients have incomplete palmar arches, which may diminish collateral perfusion, possibly leading to hand ischemia in the presence of RAO. Although rarely performed in practice, 
identifying collateral flow to the region supplied by the artery can be useful prior to puncture. While limited studies have found variable accuracy associated with such evaluations, it is believed that patients, and in particular high risk patients, undergoing radial artery puncture should have the collateral flow to the vessel evaluated(16, 17). Finger pulse plethysmography, Doppler flow measurements, and measurement of the arterial systolic pressure of the thumb have been described but are not routinely used(18).

\section{CONCLUSION:}

Radial arterial occlusion resulting in compromised circulation to the hand/fingers during the placement of an indwelling arterial catheter at the time of surgery is a rare phenomenon but has significant potential consequences. Consideration should be given to utilising a reliable test to establish patency of the palmar arch. Furthermore, all patients should be examined for radial artery patency before discharge. Novel studies and techniques are needed to improve strategies that minimise the incidence of this major complication of radial access. We describe two such cases in which timely recognition of this complication contributed to successful management, with both patients making a full recovery.

\section{Declarations:}

Conflicts of interest/Competing interests : There are no conflicts of interest to declare. There exists no financial interest or connection, direct or indirect, or other situation that might raise the question of bias in this work reported or the conclusions, implications or opinions stated - including pertinent commercial or other sources of funding for the individual author(s) or for the associated department(s) or organisation(s), personal relationships, or direct academic competition.

\section{Authorship Contributions:}

1. Dr Alison Deasy - substantial contribution to the acquisition, analysis and interpretation of data; involved in drafting the manuscript; agreed to be accountable for all aspects of the work in ensuring that questions related to the accuracy or integrity of any part of the work are appropriately investigated and resolved.

2. Dr Tanya O'Neill - substantial contribution to the acquisition, analysis and interpretation of data; involved in drafting the manuscript; gave final approval of the version to be published; agreed to be accountable for all aspects of the work in ensuring that questions related to the accuracy or integrity of any part of the work are appropriately investigated and resolved.

3. Mr Daniel Rawluk - substantial contribution to the analysis of data; involved in drafting the manuscript; gave final approval of the version to be published; agreed to be accountable for all aspects of the work in ensuring that questions related to the accuracy or integrity of any part of the work are appropriately investigated and resolved.

4. Mr Joseph Deasy - substantial contribution to the analysis of data; involved in drafting the manuscript; gave final approval of the version to be published; agreed to be accountable for all aspects of the work in ensuring that questions related to the accuracy or integrity of any part of the work are appropriately investigated and resolved.

1. Scheer B, Perel A, Pfeiffer UJ. Clinical review: complications and risk factors of peripheral arterial catheters used for haemodynamic monitoring in anaesthesia and intensive care medicine. Crit Care. 2002;6(3):199-204.

2. Bedford RF. Radial arterial function following percutaneous cannulation with 18- and 20-gauge catheters. Anesthesiology. 1977;47(1):37-9.

3. Davis FM, Stewart JM. Radial artery cannulation. A prospective study in patients undergoing cardiothoracic surgery. Br J Anaesth. 1980;52(1):41-7.

4. Onal O, Salman E, Yetisir F, Kilic M. Hand ischaemia after radial artery cannulation. BMJ Case Rep. $2015 ; 2015$. 
5. Wilkins RG. Radial artery cannulation and ischaemic damage: a review. Anaesthesia. 1985;40(9):896-9.

6. Bernat I, Aminian A, Pancholy S, Mamas M, Gaudino M, Nolan J, et al. Best Practices for the Prevention of Radial Artery Occlusion After Transradial Diagnostic Angiography and Intervention: An International Consensus Paper. JACC Cardiovasc Interv. 2019;12(22):2235-46.

7. Sanmartin M, Gomez M, Rumoroso JR, Sadaba M, Martinez M, Baz JA, et al. Interruption of blood flow during compression and radial artery occlusion after transradial catheterization. Catheter Cardiovasc Interv. 2007;70(2):185-9.

8. Pancholy S, Coppola J, Patel T, Roke-Thomas M. Prevention of radial artery occlusion-patent hemostasis evaluation trial (PROPHET study): a randomized comparison of traditional versus patency documented hemostasis after transradial catheterization. Catheter Cardiovasc Interv. 2008;72(3):335-40.

9. Cubero JM, Lombardo J, Pedrosa C, Diaz-Bejarano D, Sanchez B, Fernandez V, et al. Radial compression guided by mean artery pressure versus standard compression with a pneumatic device (RACOMAP). Catheter Cardiovasc Interv. 2009;73(4):467-72.

10. Shroff AR, Fernandez C, Vidovich MI, Rao SV, Cowley M, Bertrand OF, et al. Contemporary transradial access practices: Results of the second international survey. Catheter Cardiovasc Interv. 2019;93(7):1276-87.

11. Avdikos G, Karatasakis A, Tsoumeleas A, Lazaris E, Ziakas A, Koutouzis M. Radial artery occlusion after transradial coronary catheterization. Cardiovasc Diagn Ther. 2017;7(3):305-16.

12. Ruzsa Z, Kovacs N, Merkely B. Retrograde subintimal recanalization of a radial artery occlusion after coronary angiography using the palmar loop technique. Cardiovasc Revasc Med. 2015;16(4):259-61.

13. Babunashvili A, Dundua D. Recanalization and reuse of early occluded radial artery within 6 days after previous transradial diagnostic procedure. Catheter Cardiovasc Interv. 2011;77(4):530-6.

14. Chim H, Bakri K, Moran SL. Complications related to radial artery occlusion, radial artery harvest, and arterial lines. Hand Clin. 2015;31(1):93-100.

15. White L, Halpin A, Turner M, Wallace L. Ultrasound-guided radial artery cannulation in adult and paediatric populations: a systematic review and meta-analysis. Br J Anaesth. 2016;116(5):610-7.

16. Kohonen M, Teerenhovi O, Terho T, Laurikka J, Tarkka M. Is the Allen test reliable enough? Eur J Cardiothorac Surg. 2007;32(6):902-5.

17. Jarvis MA, Jarvis CL, Jones PR, Spyt TJ. Reliability of Allen's test in selection of patients for radial artery harvest. Ann Thorac Surg. 2000;70(4):1362-5.

18. Asif M, Sarkar PK. Three-digit Allen's test. Ann Thorac Surg. 2007;84(2):686-7. 